\title{
Trans-arterial Embolization in a Patient with Unilateral Absence of Pulmonary Artery: Treatment Success and the Four-year Prognosis
}

\author{
Yoshitaka Ono ${ }^{1}$, Kyoko Niimi ${ }^{1}$, Yukihiro Horio ${ }^{1}$, Takahisa Takihara ${ }^{1}$, Genki Takahashi ${ }^{1}$, \\ Kazuki Harada ${ }^{1}$, Hiroto Takiguchi ${ }^{1}$, Katsuyoshi Tomomatsu ${ }^{1}$, Naoki Hayama ${ }^{1}$, \\ Tsuyoshi Oguma ${ }^{1}$, Takuya Aoki ${ }^{1}$, Tetsuya Urano ${ }^{1}$, Tatsuya Sekiguchi ${ }^{2}$, Jun Koizumi ${ }^{2}$ and \\ Koichiro Asano ${ }^{1}$
}

\begin{abstract}
:
Unilateral absence of the pulmonary artery (UAPA) with or without other anomalies in the heart is a rare congenital malformation. A 55-year-old Filipino woman without a remarkable medical history was admitted to our hospital for hemoptysis. Contrast-enhanced chest computed tomography revealed the absence of the left pulmonary artery. Echocardiography and right heart catheterization showed no cardiac malformations or pulmonary hypertension. We diagnosed her with isolated left-sided UAPA and performed transarterial embolization of the left inferior phrenic artery. This resolved the hemoptysis, and there was no recurrence during the four-year follow-up period.
\end{abstract}

Key words: Angiography, Cardiovascular malformation, Embolization, Hemoptysis

(Intern Med Advance Publication)

(DOI: 10.2169/internalmedicine.8667-21)

\section{Introduction}

The prevalence of unilateral absence of pulmonary artery (UAPA) without associated cardiovascular anomalies, or isolated UAPA, ranges from 1 in 200,000 to 300,000 (1). In these cases, the blood supply to the lungs is through the systemic circulation, such as bronchial arteries, intercostal arteries, and internal thoracic arteries (2). Patients with isolated UAPA in adulthood are generally asymptomatic, although they may present with hemoptysis or repetitive respiratory infections that require specific treatments (3-6). However, there is limited information available on therapeutic approaches for isolated UAPA.

We herein report the successful treatment of a patient presenting with hemoptysis due to isolated UAPA via transarterial embolization (TAE) of the collateral artery.

\section{Case Report}

A 55-year-old Filipino woman was admitted to the hospital because of bloody sputum and hemoptysis (blood loss of approximately $70 \mathrm{~mL}$ ) in the past 2 weeks accompanied by persistent cough for 1 month. Except for untreated hypertension, she and her family had no history of cardiopulmonary diseases. Her blood pressure was $140 / 80 \mathrm{mmHg}$, heart rate was $92 / \mathrm{min}$, respiratory rate was $18 / \mathrm{min}$, and percutaneous oxygen saturation $\left(\mathrm{SpO}_{2}\right)$ was $98 \%$ under room air breathing. The left rib cage was smaller than the right, and a physical examination revealed decreased breath sounds in the left chest. No crackles were heard in the bilateral lung fields. There were no abnormalities in the peripheral blood counts or results of coagulation or blood biochemical tests, including serum brain natriuretic peptide levels.

On admission, chest radiography revealed a smaller size

${ }^{1}$ Division of Pulmonary Medicine, Department of Medicine, Japan and ${ }^{2}$ Department of Diagnostic Radiology, Tokai University School of Medicine, Japan

Received: September 13, 2021; Accepted: November 29, 2021; Advance Publication by J-STAGE: January 13, 2022

Correspondence to Dr. Kyoko Niimi, niimi_k1002@tokai-u.jp 
A

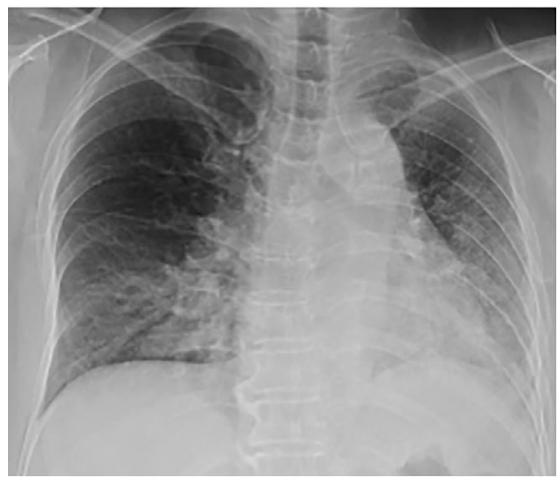

B

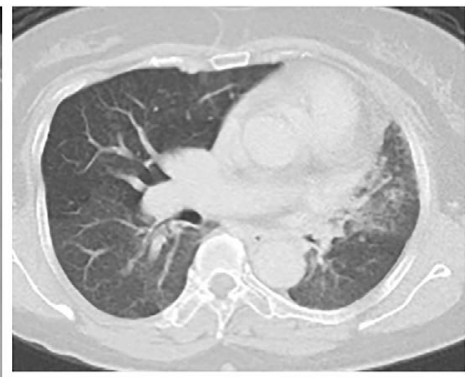

D

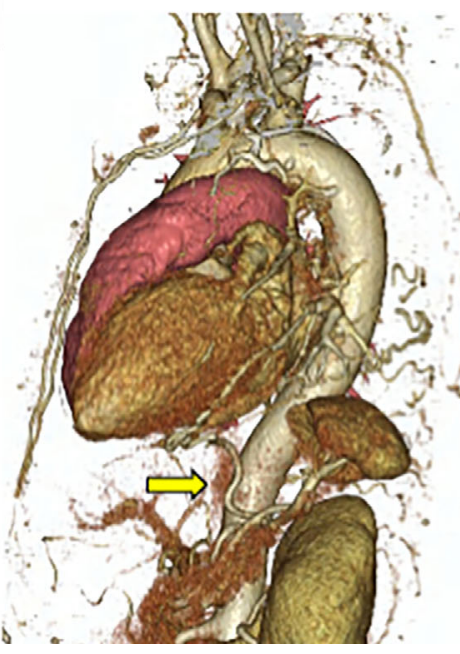

C

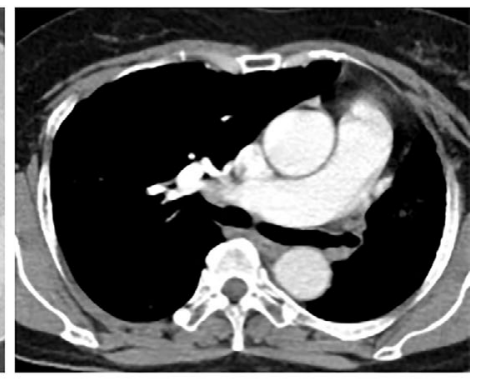

$\mathrm{E}$

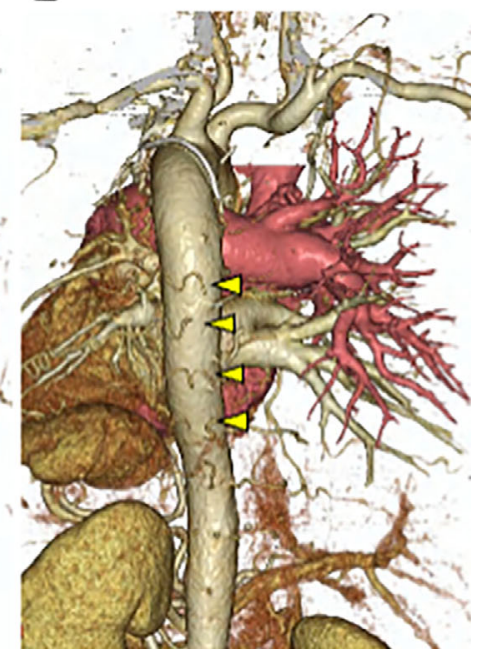

Figure 1. Radiography findings on admission. Chest radiograph (A) showing decreased volume and permeability of the left lung. Chest $\mathrm{CT}(\mathrm{B}, \mathrm{C})$ demonstrating ground-glass opacity in the lingua of the left lung and absence of the left pulmonary artery. Three-dimensional chest CT showing the dilated and tortuous left inferior phrenic artery (yellow arrow, D) and 6th-9th intercostal arteries (yellow arrow heads, E), which are running into the left inferior lung field. CT: computed tomography

of the left lung than the right, with decreased permeability (Fig. 1A). Thoracic computed tomography (CT) revealed ground-glass opacity in the lingua of the left lung (Fig. 1B), and contrast-enhanced images demonstrated that the left pulmonary artery was absent (Fig. 1C). In addition, the left inferior phrenic artery and $6^{\text {th }}-9^{\text {th }}$ intercostal arteries were dilated and tortuous, feeding on the left lower lobe of the lung (Fig. 1D, E). Selective angiography of the left inferior phrenic artery showed a contrasting effect on the left inferior lung field (Fig. 2A). Neither an early description of the pulmonary venous phase nor shunt formation was observed. Echocardiography and right heart catheterization revealed no cardiac malformations or pulmonary hypertension; the mean pulmonary arterial pressure was $21 \mathrm{mmHg}$, and the pulmonary vascular resistance was 130 dynes $/ \mathrm{s} / \mathrm{cm}^{-5}$. A bronchoscopic examination revealed no lesions that might be causing active bleeding. Therefore, we diagnosed this as a case of isolated left-sided UAPA, presuming the left inferior phrenic artery to be the vessel responsible for hemoptysis.

On day 3 of admission, TAE was performed using a gelatin sponge, which was prepared by cutting the sponge into 1-mm squares and mixing and crushing it in contrast medium via the pumping method (7-9). The contrast effect of the pulmonary field disappeared after the TAE of the left inferior phrenic artery (Fig. 2B). Her blood pressure was controlled with an oral calcium blocker. There were no episodes of hemoptysis or respiratory tract infections during the fouryear period after TAE without further intervention on the left intercostal arteries.

\section{Discussion}

Isolated UAPA is often asymptomatic, but sometimes presents with hemoptysis or recurrent respiratory tract infections. Although surgical therapy, TAE, and hemostatic materials are often considered (10-14), there has been no established treatment for symptomatic isolated UAPA. In the present case, long-term control of hemoptysis was achieved using a single TAE procedure.

There have been three reported cases of UAPA that achieved long-term control of hemoptysis with TAE: two men, 37 and 40 years old, who were treated with coil embolization (13), and a 31-year-old man for whom nonspherical polyvinyl alcohol particles were used for treatment (14). However, most patients with hemoptysis due to UAPA who are treated with TAE suffer relapse. According 
A

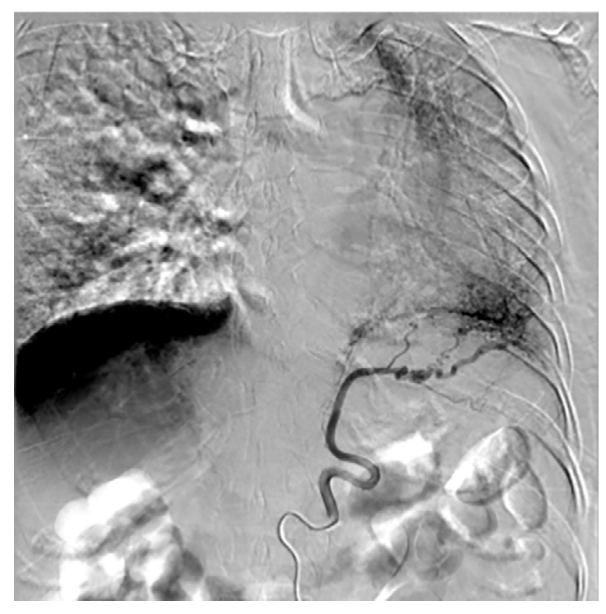

B

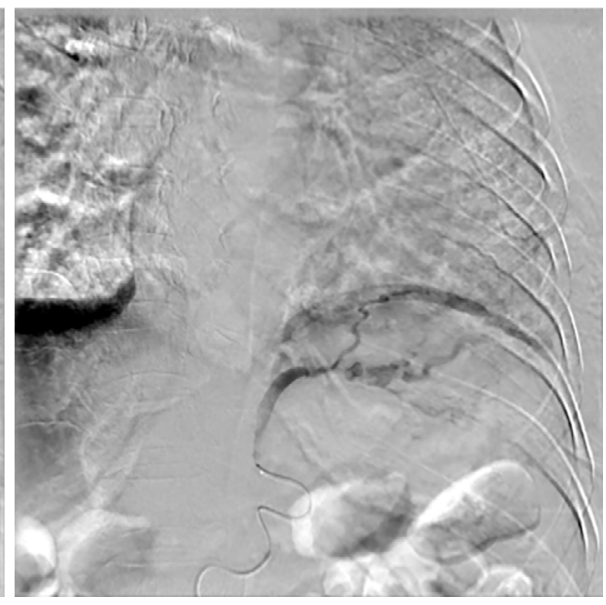

Figure 2. Angiography before and after trans-arterial embolization. (A) Selective angiography for the left inferior phrenic artery showing a stain on the left inferior pulmonary field. (B) Following embolization of left inferior phrenic artery, the abnormal stain of the pulmonary field disappeared.

to a systemic review of 65 adult patients with isolated UAPA, TAE was performed in 6 patients, and 5 of them (83\%) were eventually treated with pneumonectomy (4). These experiences suggest that treatment strategies for UAPA need to be developed with the understanding that there is a high risk of recurrence.

To successfully treat UAPA with TAE, it is important to correctly identify the responsible artery. In the present case, both the left inferior phrenic artery and intercostal arteries were dilated on contrast-enhanced CT. We therefore planned a two-stage approach-first to the left inferior phrenic artery and then to the intercostal arteries-to minimize the risk for pulmonary infarction. As the hemoptysis was completely controlled with the first embolization of the left inferior phrenic artery, a further evaluation and embolization of the intercostal arteries were not performed.

Although several materials have been used for vascular embolization, including gelatin sponge, coils, n-butyl cyanoacrylate, and polyvinyl alcohol particles, no consensus concerning the embolization materials has been reached. Coils are a powerful and permanent embolic material; however, embolization of the proximal arteries by coils may induce the development of a more distal and fragile collateral blood supply (15). N-butyl cyanoacrylate is also a permanent embolization material, but we avoided its use in this case because of the risk of infection that could have arisen due to tissue necrosis $(16,17)$. Particulate embolic materials, such as gelatin sponge and polyvinyl alcohol particles, are useful in overcoming these drawbacks. Gelatin sponge particles injected through a catheter are carried distally via the arterial bloodstream and then trapped in the vessels where blood clotting gradually takes place under the blood flow blocked by mechanical obstruction. This hemostatic ability of the gelatin sponge is almost equivalent to that of the fibrin (18). Other reports suggest that embolization with a gelatin sponge may cause permanent blockage of blood ves- sels due to the development of granulomatous vasculitis-like lesions $(19,20)$. However, temporary embolic materials have some disadvantages, such as the risk of reopening, which may lead to hemoptysis recurrence (21). In particular, when performing TAE with a gelatin sponge, it is difficult to gauge how far the catheter should be advanced toward the target and how small the embolic material should be split for administration. Thus far, the choice and use of embolic agents has been based on personal experience and expertise, with the understanding of the properties of each agent along with the anatomical and histological changes varying among cases (22).

\section{Conclusion}

We encountered a case of isolated left-sided UAPA that was successfully treated with selective TAE of the left inferior phrenic artery using a gelatin sponge, with the symptoms remaining under control for four years. There have only been a few reports concerning the long-term prognosis of UAPA after TAE, and further research is needed to establish the most appropriate treatment approach.

The authors state that they have no Conflict of Interest (COI).

\section{References}

1. Kruzliak P, Syamasundar RP, Novak M, Pechanova O, Kovacova G. Unilateral absence of pulmonary artery: pathophysiology, symptoms, diagnosis and current treatment. Arch Cardiovasc Dis 106: 448-454, 2013.

2. Ten Harkel AD, Blom NA, Ottenkamp J. Isolated unilateral absence of a pulmonary artery: a case report and review of the literature. Chest 122: 1471-1477, 2002.

3. Bouros D, Pare P, Panagou P, Tsintiris K, Siafakas N. The varied manifestation of pulmonary artery agenesis in adulthood. Chest 108: 670-676, 1995. 
4. Wang P, Yuan L, Shi J, Xu Z. Isolated unilateral absence of pulmonary artery in adulthood: a clinical analysis of 65 cases from a case series and systematic review. J Thorac Dis 9: 4988-4996, 2017.

5. Griffin N, Mansfield L, Redmond KC, et al. Imaging features of isolated unilateral pulmonary artery agenesis presenting in adulthood: a review of four cases. Clin Radiol 62: 238-244, 2007.

6. Kadir IS, Thekudan J, Dheodar A, Jones MT, Carroll KB. Congenital unilateral pulmonary artery agenesis and aspergilloma. Ann Thorac Surg 74: 2169-2171, 2002.

7. Katsumori T, Nakajima K, Mihara T, Tokuhiro M. Uterine artery embolization using gelatin sponge particles alone for symptomatic uterine fibroids: midterm results. Am J Roentgenol 178: 135-139, 2002.

8. Miyayama S, Matsui $\mathrm{O}$, Yamashiro $\mathrm{M}$, et al. Ultraselective transcatheter arterial chemoembolization with a 2 -f tip microcatheter for small hepatocellular carcinomas: relationship between local tumor recurrence and visualization of the portal vein with iodized oil. J Vasc Interv Radiol 18: 365-376, 2007.

9. Mori H, Saida Y, Watanabe Y, Irie T, Itai Y. Rapid production of gelatin sponge particles for transcatheter arterial embolization: pumping method. Nihon Igaku Hoshasen Gakkai Zasshi 60: 702704, 2000 (in Japanese).

10. Britton J, Sachithanandan A, Srinivasan L, Ghosh S. Pneumonectomy for congenital isolated unilateral pulmonary artery agenesis. Med J Malaysia 66: 363-364, 2011.

11. Bekoe S, Pellegrini RV, DiMarco RF Jr., Grant KJ, Woelfel GF. Pneumonectomy for unremitting hemoptysis in unilateral absence of pulmonary artery. Ann Thorac Surg 55: 1553-1554, 1993.

12. Shakibi JG, Rastan H, Nazarian I, Paydar M, Aryanpour I, Siassi B. Isolated unilateral absence of the pulmonary artery. Review of the world literature and guidelines for surgical repair. Jpn Heart J 19: 439-451, 1978.

13. Reñé M, Sans J, Dominguez J, Sancho C, Valldeperas J. Unilateral pulmonary artery agenesis presenting with hemoptysis: treatment by embolization of systemic collaterals. Cardiovasc Intervent Radiol 18: 251-254, 1995.
14. Dhanoa D, Jaskolka JD, Darling G, Hanna W. Particle embolization as primary endovascular management of a patient with massive hemoptysis and isolated unilateral absence of the left pulmonary artery. J Vasc Interv Radiol 22: 256-258, 2011.

15. Hu J, Albadawi $\mathrm{H}$, Chong BW, et al. Advances in biomaterials and technologies for vascular embolization. Adv Mater 31: e 1901071, 2019.

16. Chagla AS, Balasubramaniam S. Cerebral N-butyl cyanoacrylate glue-induced abscess complicating embolization. J Neurosurg 109: 347, 2008.

17. Baltacioğlu F, Cimşit NC, Bostanci K, Yüksel M, Kodalli N. Transarterial microcatheter glue embolization of the bronchial artery for life-threatening hemoptysis: technical and clinical results. Eur J Radiol 73: 380-384, 2010.

18. Jenkins HP, Janda R. Studies on the Use of Gelatin Sponge or Foam as an Hemostatic Agent in Experimental Liver Resections and Injuries to Large Veins. Ann Surg 124: 952-961, 1946.

19. Ishikura H, Sotozaki Y, Adachi H, Sato M, Yoshiki T. Granulomatous arteritis with massive eosinophilic leukocyte infiltration and transient peripheral eosinophilia subsequent to transarterial embolization therapy with a gelatin sponge. Acta Pathologica Japonica 41: 618-622, 1991.

20. Jander HP, Russinovich NA. Transcatheter gelfoam embolization in abdominal, retroperitoneal, and pelvic hemorrhage. Radiology 136: 337-344, 1980.

21. Hahn S, Kim YJ, Kwon W, Cha SW, Lee WY. Comparison of the effectiveness of embolic agents for bronchial artery embolization: gelfoam versus polyvinyl alcohol. Korean J Radiol 11: 542-546, 2010.

22. Abada HT, Golzarian J. Gelatine sponge particles: handling characteristics for endovascular use. Tech Vasc Interv Radiol 10: 257260, 2007.

The Internal Medicine is an Open Access journal distributed under the Creative Commons Attribution-NonCommercial-NoDerivatives 4.0 International License. To view the details of this license, please visit (https://creativecommons.org/licenses/ by-nc-nd/4.0/).

(C) The Japanese Society of Internal Medicine Intern Med Advance Publication 